\title{
Transporte terrestre, un tema regional estratégico del Tratado de Libre Comercio de América del Norte
}

\author{
Margarita Camarena LuHrs*
}

\section{RESUMEN}

A veinte años del Tratado de Libre Comercio de América del Norte (TLCAN), el cumplimiento y los pendientes del Capítulo xx sobre transporte siguen siendo tema de interés estratégico para sostener el aumento del comercio, cuidar la seguridad nacional, especialmente de Estados Unidos, e impulsar la integración regional con ventajas comunes para los tres países. Si para los tres socios han aumentado los flujos de comercio, para México es evidente que la construcción de cien proyectos de infraestructura de transporte para hacerlos posibles ha dejado de lado problemas de integración regional previos y han surgido nuevas desigualdades con los intentos fallidos de alcanzar la libertad de tránsito del Tratado.

Palabras clave: TLCAN , estrategia, integración, transporte, Norteamérica.

\section{ABSTRACT}

Twenty years after the North American Free Trade Agreement came into effect, compliance with and pending issues regarding Chapter $\mathrm{xx}$ about transportation continue to be a matter of strategic interest for maintaining the increase in trade, ensuring national security (especially in the United States) and fostering regional integration with common advantages for the three countries. While trade has increased for the three partners, for Mexico, it is clear that the construction of 100 transportation infrastructure projects to make that possible have left to one side previously existing problems of integration, and that new inequalities have emerged with the failed attempts to achieve the agreement's freedom of transit.

Key words: NAFTA, strategy, integration, transportation, North America.

\footnotetext{
* Investigadora del Instituto de Investigaciones Sociales, UNAM, <margarita@sociales.unam.mx>.
} 


\section{INTRODUCCIÓN ${ }^{1}$}

Después de veinte años de la entrada en vigor del TLCAN, el avance en la integración económica de Norteamérica es un hecho; especialmente si se observa el trazo de los corredores del TLCAN, el crecimiento de la infraestructura de transporte carretero y multimodal, así como el aumento en los volúmenes de tránsito y tráfico. Ese crecimiento es distinto para ciudades, puertos y regiones de cada uno de los tres países, porque la operación del transporte enfrenta aún elevados costos, demoras y obstáculos que limitan el alcance de niveles equivalentes de competitividad de los corredores transfronterizos de transporte, especialmente desde o hacia México. ${ }^{2}$

En este artículo se constata que tanto el aumento del comercio y del transporte internacional como los efectos de este crecimiento no fueron contemplados o son distintos de los previstos. Según el Capítulo xx del TLCAN, desde 1995 se debió realizar un transporte transfronterizo sin restricciones, pero hasta ahora esto sigue siendo un tema estratégico pendiente que ha dado lugar a numerosas controversias por los incumplimientos del tratado, así como a lentos avances en los acuerdos parciales, entre los que destacan los de 2011, para la apertura plena y permanente del transporte, pues hasta el momento sólo se permite el libre tráfico internacional de contenedores.

Si en efecto Norteamérica ya se ha constituido en un espacio económico, político y social que cumple una importante función en el proceso de recentramiento de la economía del mundo y han crecido significativamente las actividades comerciales, también han aumentado las desventajas y efectos perniciosos para México y algunas localidades y regiones del resto de Norteamérica, puesto que se han profundizado las desigualdades en el desarrollo y desequilibrios internos, sin alcanzarse las ventajas comunes de la libertad de tránsito de las mercancías. Quedan pendientes aspectos estratégicos y prácticos que ponen en duda la ventaja común y el éxito del proceso integrador del transporte para el TLCAN.

Con el aumento de los intercambios, se han hecho aún más evidentes los bloqueos al libre tránsito internacional. Los tiempos y costos de cruce transfronterizo y regional resultan excesivos, reduciendo la competitividad buscada. Las dificultades

\footnotetext{
${ }^{1}$ Una versión preliminar de este artículo fue presentada como ponencia en la Conferencia internacional "A 20 años del tLCAN. Viejos problemas, nuevos desafíos", organizada por la Facultad de Economía de la Universidad Nacional Autónoma de México, el 24 de enero de 2014.

${ }^{2}$ Los corredores de transporte transfronterizos del TLCAN se han identificado por itinerarios seguidos por las rutas de viaje, a través de las mallas de carreteras federales y estatales de México, Estados Unidos y Canadá, para enlazar lugares de origen y destino de los viajes realizados. Su trazo y operación unitaria permitirían aprovechar capacidades competitivas compartidas por los tres países, agilizando embarques sin transferencias y reduciendo costos; sin embargo, aún se requiere un promedio de tres camiones por viaje de productos exportados o importados, sin haberse logrado eliminar esos costos extra por cruce fronterizo, logísticos y de transporte así como de tiempo y energía.
} 
que representa el paso a través de los puntos de frontera internacional, así como las dificultades de la transferencia de la carga de unos modos de transporte a otros, el cruce y lo tortuoso de los trámites administrativos, especialmente en el transporte por carreteras entre México y Estados Unidos, hacen que México resulte estratégico como puente terrestre, a pesar de ser territorio caro, difícil y riesgoso de transitar.

Además, es desigual la continuidad territorial transfronteriza con apoyo del transporte que abriría paso a la integración comercial, laboral y jurídica según el tratado. De cierta manera hay razones históricas y geográficas en México que han facilitado la integración del Norte, pero que dificultan la del centro y sur-sureste mexicanos con el resto de Norteamérica. La nueva conectividad del país alcanza hasta el Darién, vía el Plan Puebla-Panamá, cuyos corredores se aúnan y prolongan los del TLCAN, justamente desde Puebla hasta Panamá.

En México se ha dado un impulso histórico a la construcción de carreteras y obras de infraestructura de transporte, y en cierta medida ello ha sido posible por la visión de la Subsecretaría de Infraestructura de la Secretaría de Comunicaciones y Transportes ( $\mathrm{SCT}$ ), durante el sexenio calderonista, cuando se hizo acopio de medio siglo de experiencias exitosas por los "camineros" de México, resolviendo los problemas de conectividad vertical y horizontal, no sólo concéntrica, de la red de transportes mexicana, aunque fuera para atender prioridades de conectividad extranjeras y transnacionales.

Algunas medidas parciales negociadas en el marco del TLCAN en 2011 han resuelto contradicciones seculares de la integración territorial mexicana, pero han dejado grandes temas sin atender, con lo que incluso se han acentuado las asimetrías que se pretendía resolver, especialmente para la organización económica y espacial subregional de México. Y es que han sido desatendidos mercados internos y regionales para asegurar la fluidez del tráfico que da prioridad a los movimientos de exportación e importación, a través de grandes distancias transnacionales, dejando vacíos territoriales y rompiendo articulaciones que habían sido funcionales mucho tiempo, con lo que se ha impulsado la emigración a las grandes ciudades y capitales medias de México a Estados Unidos y Canadá. Considerado este contexto, en el que parecería que el TLCAN ya cumplió con lo previsto y lo que era posible, en este trabajo se revisan aspectos del transporte por carretera que tienen importancia estratégica y económica como gran tema que tuvo que ser reiteradamente aplazado y que dejó de cumplir lo pactado con el tratado, sin alcanzarse las ventajas comunes declaradas. Lo anterior resulta crítico, especialmente porque condiciona las siguientes etapas de desarrollo interno y de alineación económica y política de Norteamérica. ${ }^{3}$

3 “Desde la firma del TLCAN en 1994, la integración de Norteamérica ha ido cambiando tanto la estabilidad macroeconómica como las estructuras territoriales internas e internacionales, y sus instituciones suprana- 
Con información estudiada en este artículo, se sugiere que el transporte y las circulaciones de Norteamérica fueron gestados por largos intercambios comerciales y culturales entre las naciones de la región, y que con el TLCAN se han profundizado muchas de esas continuidades y diferencias; sin embargo, se sugiere también que la experiencia de los últimos años podría aprovecharse, con voluntad política, para la promoción de intereses y ventajas comunes que hagan notables las ventajas de superar dichas desigualdades económicas, comerciales, culturales y de todo tipo.

Los corredores del TLCAN, construidos a la fecha, demuestran una tendencia integradora que, si es adoptada de manera conjunta y positiva, podría resultar no sólo una consecuencia irreversible de la sujeción trinacional de 1994 a la fecha, sino tener otros efectos benéficos para, primero, dar coherencia interna a las naciones participantes, y luego, sistematicidad a las vinculaciones internacionales a través de sus territorios y desde las economías motrices involucradas, en el mediano y largo plazos.

Para ello, cabe destacar el papel de México como contrapeso territorial y puente terrestre importante de las circulaciones de Norteamérica en su conjunto, y particularmente de Estados Unidos, dado que México, posiblemente junto con otras regiones desiguales y discontinuas de menores desarrollos relativos que hay en Estados Unidos y Canadá, constituye el contrapeso de los equilibrios territoriales y políticos de América del Norte. De acuerdo con ello, cabe resaltar que un espacio vecino como el mexicano, por más que se intente dejarlo así de rezagado y "libre" o vacío de atractivo a la expansión estadunidense, inevitablemente resulta arrastrado como contrapeso de la dualidad norteamericana, integradora y desintegradora, en escalas macro y microrregionales, que conmueve con toda su administración territorial al resto del mundo.

Así, en este contexto se puede notar cómo las conexiones Norte-Sur entre Canadá, Estados Unidos y México basculan en un conjunto territorial, que ya es continuo -entre otros factores porque sus circulaciones ya lo son y cada vez adquieren mayor regularidad, aunque sea con una intensidad y un volumen irracionales- porque se han ido acercando y estrechando cada vez más los tiempos, las distancias de los intercambios comerciales y de todo tipo, que se han asumido como efectos de complementación y diversificación de las corrientes de circulaciones de largas distancias transoceánicas de Asia-Pacífico hacia Norteamérica.

Debido a que tales corrientes son conducidas preferentemente hacia el territorio de Estados Unidos -aunque se ha encontrado que pueden ser reducidas si no en

cionales seguirán condicionando las siguientes etapas de alineación económica y política. En ese proceso, México cumple el doble papel de contrapeso territorial y de puente terrestre, pues los corredores comerciales han ido abriendo paso a otras pautas espaciales de integración económica de la producción y los mercados, que alterna la organización económica-espacial interna de las ciudad y regiones, así como de las relaciones externas de los tres países" (Camarena, 2011: 99). 
distancias y tiempos, sí significativamente por menores costos de los fletes- se ilustra con cierto detalle el efecto que ha tenido esta relocalización de empleos e inversiones de Estados Unidos hacia México, como parte de la incesante búsqueda de mayores rendimientos de las inversiones que han acentuado la vocación asignada a México como puente terrestre. A partir de esta función de puente, México actualiza su importancia geoestratégica mundial aunque se lo ha obligado, innecesariamente, a restar peso e importancia al transporte y a las circulaciones alimentadoras destinadas a satisfacer los tráficos internos de carácter regional y secundario, forzando la desarticulación de mercados regionales y microrregionales y su exclusión o integración subordinada a otras órbitas del comercio transcontinental. ${ }^{4}$

\section{Contexto de LA INTEGRACIÓN DE NoRTeAmÉRICA POR EL TLCAN}

La integración de Norteamérica ha ido cambiando desde la firma del TLCAN en 1994, a tal grado que una mayor estabilidad macroeconómica y la solidez de instituciones supranacionales parecerían condicionar las siguientes etapas de alineación económica y política en el siglo xxI; ${ }^{5}$ sin embargo, algunos antecedentes de ese largo proceso de integración económica de Norteamérica inciden en la situación actual, por ejemplo México cumple ahora el doble papel de contrapeso territorial, por ser el país de menor desarrollo e intensidad económica del subcontinente, y de puente terrestre para la internación a Estados Unidos y Canadá de un gran movimiento de flujos comerciales provenientes de Asia, así como por los menores costos salariales que, por ejemplo, reducen hasta el 15 por ciento de los costos totales de transporte por otras rutas alternativas al tráfico proveniente del Pacífico.

\footnotetext{
${ }^{4}$ Las carreteras que del este-oeste a través de los territorios de México, Estados Unidos y Canadá conectando el océano Pacífico con el Atlántico se corresponden con las carreteras longitudinales norte-sur, pero desde 2006 prolongan esa malla de manera continua a través de México, integrándolo funcionalmente. Es reciente la construcción de cuatro grandes ejes de carreteras transversales (Mazatlán-Tamaulipas, Colima-Tamaulipas, Acapulco-Veracruz y Salina Cruz-Coatzacoalcos), que conectan a México entre sí, y con respecto del centro del país de manera radial, pero particularmente hacia el Norte, con Estados Unidos.

Estos puentes terrestres abren pasos alternativos al gran tráfico comercial del Oriente y de los propios Estados Unidos, desde México y le imponen otra coherencia regional interna a nuestro país, precisamente con los recientemente concluidos corredores del TLCAN, que inauguran otra práctica de la integración geográfico-estratégica del Norte que sujetará a México, todavía más, aunque por lo pronto simplemente lo atraviese de manera ininterrumpida.

5 “El futuro de la integración económica regional del TLCAN dependerá de condiciones macroeconómicas nacionales estables y de la voluntad política de las partes. Para profundizar el proceso, la única alternativa, de acuerdo con la experiencia europea, es la construcción de la unión aduanera de América del Norte; sin embargo, esto no será suficiente ya que se requerirán marcos institucionales supranacionales más fuertes, como los desarrollados en Europa" (Ramírez Acosta y Castillo Ponce, 2009: s/p).
} 
Desde la firma del Tratado, los corredores comerciales han ido abriendo paso a otras pautas espaciales de integración económica de la producción y de los mercados que alteran la organización económica-espacial interna de las ciudades, los puertos y las regiones, así como de las relaciones externas de los tres países. En todo ello, cumplen funciones decisivas los movimientos de personas, bienes, capitales e informaciones de unos lugares a otros porque ordenan los espacios interiores de las ciudades y jerarquizan sus interrelaciones con otros sitios inmediatos o distantes.

Dado que en México, en la última década se ha dado un impulso a la construcción de carreteras mayor que el registrado desde 1928, sus posibilidades de integración y competitividad -en su interior y con respecto al resto de Norteamérica- son distintas porque ya son mayores los tramos carreteros concluidos, lo que ha posibilitado cerrar circuitos, enlazar corredores urbanos y regionales, conectar transversalmente las costas del Pacífico y del golfo de México y unir los pasos fronterizos del norte y el sur del país, sobre todo porque con las reducciones de costos, tiempos y distancias de recorridos se comunican más rápidamente los grandes centros de comercio, localizados en ciudades, puertos y regiones, incrementando ganancias con esa cobertura mayor y accesibilidad expedita, al mismo tiempo que la infraestructura y operación del transporte se abren al sector privado y a los negocios financieros, lo cual reduce las enormes brechas de capacidades logísticas y de transporte de México respecto de Estados Unidos y Canadá. ${ }^{6}$

Circulaciones irregulares que son completadas o incorporadas por las nuevas redes carreteras y de transporte multimodal adquieren capacidades de coherencia geoestrategia puntual y global, en el contexto de toda Norteamérica, cambiando la estructura y las funciones espaciales de interconexión urbana y regional, local e internacional que había en México hasta hace poco más de una década.

Estos efectos en la estructuración espacial multiplican las facilidades de intercambio pero profundizan las asimetrías espaciales y las desigualdades socioeconómicas, puesto que las áreas y los puntos enlazados son solamente los de largos recorridos que resultan de mayor interés, por el mayor volumen y la rentabilidad de lo transportado o por las seguridades y fortalezas geoestratégicas que representan en el concierto mundial de la entrada-salida de Estados Unidos.

\footnotetext{
${ }^{6}$ Esto tiene múltiples consecuencias, dos muy importantes son que, por una parte, se "aprecia" la inversión pública en carreteras que eran patrimonio de la nación y ahora son parte de los activos de algunas transnacionales de la construcción o las finanzas, y por otra, esos costos "descontados" o no transferidos al costo total de las mercancías transportadas ahora sí se transfieren al consumidor final directamente, aumentando todos los costos y convirtiendo a la producción de transportes en un doble campo especulativo financiero, como actividad productiva y como "llave" dosificadora del abasto, controlando por las dos vías la velocidad del dinero y las pautas sociales de consumo.
} 
$\mathrm{Al}$ acelerarse las circulaciones económicas, el aumento de las relaciones internas e interurbanas de las economías que expresan posiblemente se amplían con los nuevos destinos y distancias accesibles. Cambian los alcances de todo tipo de intercambios, pero al mismo tiempo se eliminan vastos territorios, regiones y ciudades que no alcanzan los volúmenes de tráfico, las escalas de desplazamientos o los grados de competitividad y ritmos expansivos de esas nuevas redes de coherencia supraterritorial.

Superavenidas sectoriales y selectivamente localizadas para los fines de la interacción global de ciertas mercancías reflejan el curso de las inversiones y los ritmos de las rentabilidades financieras que marcan los niveles de ganancias que atraen o eliminan capitales, introduciendo pautas discriminatorias entre las propias fracciones de clase y tipos de capitales movilizados transnacionalmente, lo cual es más grave, pues esta exclusión limita o fractura de lleno las posibilidades de los incipientes o aislados mercados locales y regionales, condenándolos a dinámicas de desalojo y vacío espacial en todos sentidos.

Este proceso interno y externo de expansión de los transportes y las circulaciones responde a la lógica global del capitalismo desde la década de 1980 y especialmente en la actualidad. Si esta expansión económica y territorial propicia mayor rapidez, cantidad y alcance de las circulaciones, y es impulsada por las nuevas tecnologías de la información y la comunicación (TIC), que subyace al impulso de mejoras logísticas y en la complementariedad de los transportes, en México esa nueva continuidad física y funcional de las carreteras -ahora enlazables de Norte a Sur y entre el Pacífico y el golfo de México- define otra geografía económica y cultural y, con ello, otra estrategia de acceso y conectividad territorial, comercial, geográfica y política, que sucede tanto en lo interno como en sus prolongaciones hacia el resto de Norteamérica, con consecuencias inmediatas constatables pero de predicciones inciertas a mediano y largo plazos. ${ }^{7}$

El hilo conductor de estas observaciones es la orientación geoestratégica de la infraestructura de transportes, por carreteras, y de lo que circula por ellas en México, en especial desde 2007, cuando se completa la construcción de diez de los catorce corredores de transporte por carretera, longitudinales y transversales, que ahora ya conectan de manera continua a las ciudades y regiones del país entre sí y con el resto de Norteamérica.

\footnotetext{
7 “En este contexto, la tendencia debe ser generar, paulatinamente, más y mejores análisis científicos que garanticen las condiciones para una integración económica regional más profunda, que debe ir más allá del acuerdo comercial hacia una etapa de alineación arancelaria como preludio para la formación de una unión aduanera en América del Norte. Desde esta perspectiva, la integración económica regional en América de Norte deberá ser lenta pero inexorable e irreversible" (Ramírez Acosta y Castillo, 2009: s/p).
} 
De igual modo, se pretende mostrar que el comportamiento de los flujos de transporte, cada vez más regulares y continuos, que forman sistemas de redes integran a las circulaciones de México con el resto de Norteamérica -especialmente desde la gran transición urbana de México en los años setenta y con la firma del TLCAN-, ya empiezan a tener, quizá desde 2003, otra presencia y otro peso de la economía mexicana como contrapeso del resto del subcontinente y como territorio de reserva estratégico en la reconducción mundial de los grandes flujos de comercio, dada su participación como puente terrestre de nuevas corrientes de la economía mundial.

Otros horizontes de integración, ahora quizá ya irreversibles, se han sucedido en los vuelcos de la economía estadunidense y canadiense del Este al Oeste y del Norte al Sur, que en este siglo voltean nuevamente hacia las costas del Atlántico y que se prolongan territorialmente, con toda intención desde el 2003, hacia México, tal como se muestra con lo que ha sucedido a la integración fronteriza de los doce puntos de ingreso y salida más importantes del norte de México, así como con la incorporación de sectores de actividad completos en las ciudades y yacimientos naturales que ya desde los años setenta dejaron atrás la traza y estructura nodal de las capitales mineras y coloniales del país, como Zacatecas, Pachuca, Taxco, San Luis Potosí, Guanajuato, que serán reemplazadas por los nuevos centros urbanos manufactureros.

Entre 2007 y 2012 culmina una larga etapa del esfuerzo geoestratégico integrador del territorio nacional cuya historia ha estado marcada por las luchas por la identidad de grupos sociales, comunidades y regiones desde mucho tiempo atrás y por los procesos de conformación del Estado nacional, característicos del siglo xx y que aún no han concluido en México.

Este reemplazo progresivo de coherencias espaciales, con otras ciudades y otras delimitaciones de interés geoestratégico territorial, constituye un gran mosaico del proceso de integración y alineación de Norteamérica en curso. Ante esta circunstancia hace falta una coherencia sectorial y espacial que dé sentido a inercias y tendencias urbanizadoras, de acuerdo con el proyecto de nación, que anime al país para que se asigne una intencionalidad al vuelco territorial hacia Norteamérica, pues todavía no es claro en qué beneficiaría a México.

Ciudades y regiones, sus transportes carreteros y sus circulaciones integradas ahora por otras coherencias transfronterizas y transnacionales, siguen reduciendo la autonomía territorial y la soberanía nacional, en la medida en que avanza la integración y la alineación de las ciudades y regiones principales, sobre todo del Norte, con el resto de regiones y sectores de las economías de América del Norte. 


\section{Tendencias a la INTEGRACIÓn Del tRANSPORTE De NoRTEamÉRICA}

El crecimiento demográfico, la producción y los intercambios comerciales entre los países y regiones de Norteamérica indican una tendencia hacia la mayor integración del subcontinente, sólo que para que ocurra aún habrán de superarse asimetrías y desafíos estructurales que hagan posible avanzar hacia otras mejores formas de agrupamiento y cohesión supraterritorial basadas en la cooperación, la equidad y el desarrollo regional sustentable, compartido en ventaja común de toda Norteamérica, aunque ello esté lejos de las intenciones prácticas de los gobiernos y poderosos grupos de interés.

Mientras tanto, hay fuertes evidencias de que ya existe una interrelación económica y espacial, la cual ha sido posible por las facilidades de transporte y circulación con que se cuenta. ${ }^{8}$ Debido a que con el aumento en la intensidad de las actividades, promovida por el capital financiero mundial desde 1980, los transportes son una contraparte flexibilizadora de los espacios urbanos, fijos y rígidos, centralizadores que son las ciudades, a las que sirven contradictoriamente, porque el control sobre transportes y circulaciones que ejerce este sector del capital efectivamente agiliza los circuitos económicos y, con ellos, acorta los ciclos de inversión, intensifica la apreciación de los valores y regula los tiempos-lugares de la realización del capital expandido, por la venta y el consumo de mercancías.

Con ello, el control de las circulaciones físicas, así como de las informaciones, se anticipa pero al mismo tiempo obstaculiza y frena los ritmos de expansión de capitales, mercados, consumos, por la falta de conexión temporal y regional de su operación, planeada con una racionalidad integrada e intencionada más allá de las ganancias que sí atienda a la coherencia y consecuencia sobre el resto de la vida económica y espacial de las sociedades a las que habrían de servir.

Como los transportes y las comunicaciones agilizan la vida de las ciudades haciendo posible la circulación de bienes y el acceso de personas que no se pueden encontrar al mismo tiempo en los mismos lugares, pero haciéndolo así se llega a niveles de saturación irracional evidente en los frecuentes cuellos de botella, el congestionamiento del tráfico y tránsito muestra cómo la vida actual depende del éxito o fracaso del traslado de lo que mueve a las economías y regiones de los tres países, sin pérdidas de tiempo.

\footnotetext{
${ }^{8}$ Las circulaciones irregulares o en redes provocan de todas maneras efectos ordenadores en la organización espacial en su conjunto, naturalmente que se pueden intencionar mejor para fines planificadores del territorio, si son diseñadas de antemano. Si son utilizadas con propósitos territoriales definidos, por ejemplo, de expansión o contracción, control o apertura del suelo local o regional, incluso transnacional, se vuelven recursos geoestratégicos inevitables de la acción pública y corporativa.
} 
Los movimientos de personas, mercancías, capitales e informaciones de Norteamérica expresan esta tendencia integradora y estandarizadora de las relaciones entre los lugares, pero de manera sectorial y selectiva, no integral ni coherente. Ésta todavía provoca asimetrías, sucede sin dirigirse a fines y plazos mayores que los dictados inercialmente por mercados que se sirven de sistemas de distribución eficaz para circuitos de mercancías singularizadas basados en el transporte, con los que se dejan de lado efectos y también ventajas que acompañan otros propósitos de expansión territorial, comercial o de control de las circulaciones. ${ }^{9}$

México es una economía y un territorio que equilibra fuerzas espaciales de toda Norteamérica, dándoles cierta estabilidad al neutralizar su desmedido peso, no como lastre, sino como reserva territorial que está empezando a adquirir un papel más activo en ese sistema. Para apreciar esa capacidades del subcontinente se presentan enseguida algunos indicadores del comercio exterior, la población total y urbana, el PIB general y del transporte/ almacenaje; así como de la extensión de las redes carreteras y los volúmenes del parque vehicular de México, Estados Unidos y Canadá, los cuales sirven como contexto a estas observaciones del cambio en la estrategia territorial del subcontinente.

De 2006 a 2010, aumenta el comercio exterior de Estados Unidos y Canadá con México, el resto de los intercambios de importaciones y exportaciones entre los países de Norteamérica disminuye. No obstante la gravedad de la crisis iniciada en 2007, la masa de dinero en movimiento permanece casi igual, pues en 2006, el comercio exterior de Norteamérica sumó 1794307 millones de dólares y en el 2010, alcanzó 1786417 millones de dólares estadunidenses a precios constantes (NATS, 2011f).

Con base en datos de la agencia North American Transportation Statistics (NATS) y del Instituto Nacional de Estadística y Geografía (INEGI), la población de Norteamérica en 2010 era de 455.7 millones, distribuida de la siguiente manera: México, 112.3 millones; Estados Unidos, 309.3 millones y Canadá, 34.1 millones de personas. Puede tomarse en cuenta que el ritmo de crecimiento de esta población creció en promedio 27.7 por ciento de 1990 a 2010, pero esta tendencia, que disminuye el ritmo de aumento de la población de 2000 a 2010, parece afectar poco los crecimientos potenciales de producción o de consumo a mediano plazo, aunque haya consecuencias en los mercados de trabajo y de consumo general y con respecto de las variaciones de los flujos de transporte (NATS, 2011a; INEGI, 2011).

\footnotetext{
${ }^{9}$ Las circulaciones ordenan los espacios edificados y jerarquizan sus relaciones. Ya sea que se trate de movimientos espontáneos, inerciales o bien, intencionales y premeditados, ya sea que conformen flujos que sí cuenten con infraestructura y normas de altas especificaciones o que por el contrario no dispongan de estas condiciones regulares para realizar tránsitos y tráficos regulares y repetitivos, el resultado de los cambios en la posición espacial del conjunto de todos estos desplazamientos en los espacios trinacionales de México, Estados Unidos y Canadá ordena y organiza sus espacios nacionales y sus relaciones internas y externas.
} 
En contraste, el PIB de los tres países aumenta entre el 2000 y hasta el 2007. Se duplica de 2000 a 2008 en Canadá y en Estados Unidos y México crece entre el 40 y el 50 por ciento. Esto demuestra que la economía crece más rápido que la población, lo cual pronosticaría una tendencia de bonanza y mejora de la calidad de vida, pero este mayor ritmo del crecimiento de la producción -y con ella de los excedentes- se traduce, por el contrario, en la segunda crisis mundial más grave de la historia mundial.

Sin embargo, es paradójico el crecimiento del PIB del transporte de los tres países, el cual es muy superior al promedio general de cada una de estas economías. En 2008 el PIB del transporte de México fue de 69.6 mil millones de dólares, el de Estados Unidos, casi seis veces mayor al alcanzar 41.6 mil millones de dólares y el de Canadá, de 61.8 mil de millones, pero en relación con un PIB general de la economía, superior al de México en una tercera parte, según datos a precios corrientes de 2008 (NATs, 2011g).

Por el comportamiento del tráfico, se puede notar que el ritmo de las ventas nacionales e internacionales es todavía superior al aumento del PIB, sugiriendo que el tráfico de mercancías y la intensidad del consumo, que también es mayor, han acelerado transportes y circulaciones en toda Norteamérica, aunque la masa de capitales permanezca en niveles semejantes al cabo de los ciclos de producción y consumo.

Es paradójico que la crisis financiera mundial, que se expresa singularmente en Norteamérica desde el 2008, pueda deberse a mayores excedentes obtenidos. Si se contempla la tendencia al aumento del tráfico en América del Norte, el gran aumento de 1990 al 2000, del 30 al 40 por ciento, reduce su ritmo de crecimiento con el estallido de la crisis, siete años después sólo crece un 8 por ciento, pero la intensidad de los intercambios pudieran ser un poco mayores, si se contemplan los aumentos de toneladas desplazadas por kilómetro en Norteamérica, las cuales pasaron de 10063.4 en 1990 a 13829.8 en 2000, y con una disminución en la tendencia al alcanzar 14693.6 toneladas/ kilómetros en 2007. Este indicador de rendimientos y competitividad del transporte ubicó a México en el 2007 en un lugar veinticuatro veces menor que el de Estados Unidos, con 274.7 toneladas/ kilómetro frente a las 6693.9 toneladas / kilómetro realizadas por Estados Unidos (NATS, 2011b).

Por otra parte, al observar la distribución de infraestructura carretera y del parque vehicular en los países norteamericanos, puede tenerse una idea más clara de las diferencias de capacidades comerciales que afectan su competitividad y posibilidades de alineación industrial y comercial. Juntos, Canadá y México, con 1457900 y 366096 kilómetros de carreteras, respectivamente, tienen una red de carreteras menor a la tercera parte de la de Estados Unidos, que alcanzó en 20086531276 kilómetros. Pero México, con esta infraestructura carretera de la que menos de la décima parte es de altas especificaciones, tan sólo representa el 4.38 por ciento de la red total del subcontinente, describiendo así, con toda claridad, la desigualdad de sus condiciones de comercio. 
Hay que observar la infraestructura carretera, la cual es indicativa de las capacidades de comercio, junto con los movimientos de los 306002680 vehículos de transporte carretero que las usan, según datos de 2008. De esta flota vehicular que transita en 2008, en las carreteras de México están registrados 29287903 vehículos, en Estados Unidos, 255917664 y en Canadá, 20797113 (NATS, 2011d). Puede destacarse que Canadá es más eficiente en el uso y rendimiento de sus vehículos por kilómetro aun tomando en cuenta que sus recorridos son de distancias comparativamente mucho mayores que las de los otros dos países.

\section{MÉxICO, CONTRAPeso teRRItorial de Norteamérica y puente terRestre del Pacífico y Estados Unidos}

A partir de la firma del TLCAN, el intercambio comercial trilateral se intensificó, significando para México más de trescientos billones de dólares en la actualidad, hasta que Estados Unidos alcanzó en 2010 un volumen de exportaciones de alrededor de 176537 millones de dólares, 10.3 por ciento y tercer lugar en origen de las importaciones del país vecino. Las importaciones de México de Estados Unidos, en 2010, fueron de 128998 millones de dólares, el 11.7 por ciento de las exportaciones totales de mercancías, pero el segundo lugar de destino comercial de Estados Unidos (García y Cuan, 2010).

El 70 por ciento de este intercambio comercial se efectúa por medio del autotransporte; el 8.4 por los ferrocarriles, mientras que el 16.4 por vía marítima y el 3.9 restante por otros medios de transporte. Además, puede tomarse en cuenta que el 98 por ciento de los movimientos de pasajeros se realizan por vía terrestre. “En 2008, cruzaron la frontera hacia EUA cerca de 203 millones de pasajeros, 4.9 millones de camiones de carga y cerca de 79 millones de vehículos" (García y Cuan, 2010).

De acuerdo con las tendencias descritas y según la vocación de puente terrestre que México está adquiriendo, parece evidente que persisten las tendencias hacia la liberalización de servicios de transporte de carga, dada la orientación de los proyectos económicos trinacionales hacia la apertura comercial y la integración económica de Norteamérica.

Si es posible que estas grandes tendencias ya se encontraran en curso, quizá desde principios de 1980 -especialmente con la firma del Tratado entre Canadá y Estados Unidos desde octubre de 1988-, cabe destacar que entonces como ahora, resulta indispensable dar continuidad internacional, de manera eficiente y rápida, al flujo de las mercancías que incesante ya se abría paso reconfigurando los modelos de desarrollo y con ellos, del trazo, los accesos y conexiones desde y hacia las principales ciudades, puertos y mercados de toda Norteamérica. 
Desde estos requerimientos resulta más clara la importancia económica y estratégica que adquiere México para lograr integrar el transporte con el TLCAN. Del transporte sigue dependiendo intensificar y dotar de una nueva celeridad a los flujos de carga. Y si con las directrices del capital financiero se orquestan todos los demás flujos de capitales e informaciones, incluso de personas, atendiendo las necesidades de relocalización constante de inversiones y empleos hacia las plazas que ofrecen mayores rentabilidades, ${ }^{10}$ entonces el control logístico y del transporte hace las veces de contraparte práctica de esa relativamente nueva, pero ahora indudablemente predominante, intencionalidad financiera de normalizar las ganancias extraordinarias por todas las vías posibles.

Sin embargo, la incapacidad de instrumentar dichos compromisos ha llevado a una integración superficial en la que los bienes circulan, pero no a la velocidad ni con la competitividad requerida, lo que se ha hecho evidente con el mayor costo de la logística / transporte en México, que ha venido reduciendo sectorial y selectivamente la velocidad de circulación de capitales y mercancías, de modo que no se recuperan las inversiones y los costos de lo invertido en la producción de las mercancías, y se hace más lenta la realización de las inversiones en detrimento importante de las ganancias y sobre todo de la competitividad de México, y en general de los países y regiones con menores capacidades de transporte y circulación (Camarena, 2012: 3).

Aunque desde el 2001 un panel del TLCAN emitió un dictamen favorable a México por el incumplimiento de Estados Unidos en esa materia, y aun cuando a partir de marzo de 2009 aplican medidas de represalias comerciales, dicha solución distó de ser la óptima y sólo pudo ser una forma de presionar a los socios para sentarse a la mesa a cumplir con sus compromisos que, para efectos prácticos, han sido letra muerta. Ello ha implicado que los costos por los servicios de logística entre los que se incluyen predominantemente los costos de transporte sean más elevados en el comercio bi y trilateral y que estas actividades muestren graves ineficiencias.

La apertura del transporte contribuiría a promover y hacer más eficientes los flujos comerciales desde México hacia Estados Unidos y Canadá, pero para ello se requería decisión política de los gobiernos de cada país para enfrentar a sectores que se oponen frontalmente a cualquier competencia del exterior. México intentó aprovechar las reuniones de ministros de Comercio y Economía para impulsar una solución integral y de largo plazo a la liberalización de los servicios de transporte terrestre de

\footnotetext{
${ }^{10} \mathrm{Tal}$ como empezó a hacerse evidente con la presencia de China, Brasil e India en el mercado mundial, al triplicar su presencia desde el 2000, la economía mexicana, que logró a principios de siglo cierto crecimiento, desde 2006 muestra una vulnerabilidad y debilidad mayores y en la actualidad resulta mucho más frágil que entonces, al irse reduciendo en más de una quinta parte su participación en el comercio mundial y desplazarse drásticamente su competitividad internacional.
} 
carga, pues esto era claramente necesario para abatir costos y elevar la competitividad de las exportaciones del país.

Sin embargo, a veinte años del TLCAN, aunque haya aumentado el intercambio comercial y quizá se observen momentos de mayor intensidad en la tendencia histórica de la actividad económica trinacional, es evidente que sobre todo se ha creado una plataforma para la expansión del capital financiero, sobreimpuesta a los procesos industriales, comerciales y al resto de los sectores de la actividad económica.

También puede haber coincidencia al observar que construir la infraestructura de transporte para que se integraran mutuamente las economías nacionales ha estado en el interés de importantes sectores financieros, industriales y comerciales de los tres países, sólo que contar con transporte terrestre de carga más eficiente ha sido una intención práctica que se ha cumplido más que por medio de la inversión pública de los gobiernos, por otras vías paralelas, como la creación de filiales de empresas de logística y transportistas en los tres países para dar continuidad al tráfico transnacional. ${ }^{11}$

\section{EL TRANSPORTE TERRESTRE, GRAN PENDIENTE DEL TLCAN}

Como los costos de logística y transporte son importantes dentro del costo total de comercio, en el caso de algunos productos son los más importantes y, muy por arriba de los aranceles, las variaciones en los costos de transporte tienen efectos decisivos en la competitividad internacional y en los ritmos de circulación de los capitales. De ahí que para todos los exportadores o importadores que se sirven de México como territorio de tránsito o como mercado de origen o destino final, utilizándolo como puente terrestre del tráfico que viene del Pacífico transitando hacia Estados Unidos y Canadá, resulte evidente la urgencia de resolver este tema de los bloqueos al libre tránsito de mercancías.

Además, cabe reiterar que el TLCAN, aunque se haya convertido en la principal política de los sucesivos gobiernos de México de los últimos veinte años, en realidad forma parte de procesos de mucho mayor alcance mundial, pues la expansión del capital, en su búsqueda incesante de trabajo barato para elevar las ganancias, ha

\footnotetext{
11 “El objetivo de la construcción y modernización de la nueva malla de corredores de transporte que es incrementar la productividad económica del país, para disminuir los desequilibrios regionales y el aislamientos de las comunidades rurales, así como contribuir a la generación de empleos y al impulso al desarrollo regional, se traduce en la apertura de una oportunidad para el financiamiento de la economía nacional que incluye hasta la nueva definición geoestratégica del territorio y la acelerada transición de las condiciones económicas internas aún en el contexto de graves dificultades porque el presupuesto asignado por el Congreso y las cantidades financiadas por el sector privado resultan insuficientes para cubrir las dimensiones de los proyectos de infraestructura por desarrollar" (Camarena, 2013: 14).
} 
alterado notablemente los flujos del transporte en toda Norteamérica, contribuyendo a que la debilidad del Estado mexicano haga que el país se pliegue aún más a prioridades de rentabilidad y acumulación extranjeras, no siendo excepción lo hecho en transportes.

Así, dado que cada vez son más importantes las oportunidades de ganancias extraordinarias obtenidas desde los márgenes de los procesos capitalistas, márgenes incluso territoriales, como se ha mencionado, el abaratamiento de costos por transporte y localización ha ido adquiriendo un peso más destacado, no solamente en la composición de los costos y optimización de los procesos productivos ajustados a las masas de ganancias y ritmos de acumulación financiera del capital, sino como factor que acelera o atenúa el ritmo del ciclo económico, forzado a veces brutalmente por fuerzas financieras que "dosifican" especulativamente el acceso de materias primas y productos terminados a los mercados, tanto como "regulan" a los consumidores a través del control del abasto, la distribución y, así controlan los intercambios económicos que son apoyados con los transportes y circulaciones existentes.

Estas diferencias en los componentes de los costos afectan la velocidad de la realización de las inversiones/ventas, restan capacidad de cooperación e integración comercial con lo que se agudiza el rezago y la desigualdad de la competencia entre ambos países. Además repercute en los tamaños y rotaciones de los inventarios, que en México requieren ser mayores, entre el 46 y el 58 por ciento de la misma razón inventarios / materias primas, que para Estados Unidos, lo que sobrecarga y resta agilidad a las operaciones manufactureras, dificulta los encadenamientos horizontales y verticales de los sectores y regiones de actividad, lo que repercute en la menor competitividad de México en el marco global. ${ }^{12}$

Por esto, para México es muy importante resolver el tema del transporte, especialmente el carretero, el cual mueve más del 60 por ciento de la carga y alrededor del 75 por ciento de las exportaciones mexicanas a Estados Unidos, pero además existen diversos intereses y grados de compromiso para lograr una integración mutuamente conveniente para los tres países firmantes del TLCAN. ${ }^{13}$

12 “De acuerdo con el estudio Doing Business del Banco Mundial en México los costos de logística se ubican en un rango del 15 al 20 por ciento como proporción del PIB lo que contrasta con un 9.5 por ciento de Estados Unidos, un 9 por ciento de los países de la OCDE y un 8.5 por ciento de Singapur... Por otra parte, mientras los negocios en Estados Unidos mantienen inventarios de aproximadamente el 15 por ciento del PIB, en Latinoamérica es a menudo el doble. Por ejemplo, la razón del inventario de materias primas de México es 1.58 veces, en promedio, superior al de Estados Unidos y 1.46 veces superior para bienes finales" (De la Mora, 2011:1).

13 “Para México resulta urgente resolver este tema pues el transporte terrestre de carga es el modo de prestación más importante al representar el 60 por ciento de la carga. Alrededor del 75 por ciento de las exportaciones mexicanas a los Estados Unidos se realizan por carretera y pasan por pasos fronterizos ubicados en Texas y Tamaulipas (Nuevo Laredo/Laredo), Texas y Chihuahua (Ciudad Juárez/El Paso), Arizona y Sonora (Nogales/Nogales), California y Baja California (San Diego/Tijuana). Hoy enviar una mercancía de México 
Puede insistirse en que en este contexto se han ido sucediendo incesantes crisis, haciendo más frecuentes y rápidos los cambios de localización de capitales y empleos. De igual manera resultan cada vez más buscadas las oportunidades de ganancias extraordinarias por el control logístico, y especulativo, financiero, del abasto de insumos y de la distribución internacional de productos, que se gradúa controlando ritmos y velocidades de transporte.

Así, se tiene el caso de relocalización de capitales y empleos en ambos lados de las fronteras, que con cifras publicadas en 2006, cuando comienza a intensificarse el uso del puente terrestre de México -conectando orígenes y destinos del océano Pacífico con tierra adentro de Estados Unidos-, por medio de transporte que desplaza las cargas 2000 millas, o sea el 30 por ciento del recorrido total, más al sur de Long Beach y de San Diego, para entrar por el puerto mexicano de Lázaro Cárdenas, ofrece a los usuarios exportadores un 15 por ciento de ahorro en los costos de transporte marítimo, carretero y ferroviario combinados, haciendo que el tráfico de mercancías baratas del Oriente hacia Norteamérica, especialmente a Estados Unidos y Canadá, pase por México. ${ }^{14}$

En este caso se requirió relocalizar capitales y empleos a México. Se desemplearon 750000 transportistas en California, volviéndolos a emplear en México (Vogel, 2006: 7). Para aquilatar este hecho, cabe tomar en cuenta que el flujo de contenedores que ingresa por los puertos del Pacífico mexicano había aumentado de 1994 al 2005, más del 450 por ciento (Vogel, 2006: 8).

La intensidad del flujo de contenedores es la mayor por su crecimiento y la más significativa por los tipos de contenidos de las cargas transportadas y sus rentabilidades. Con lo ilustrado por esta relocalización de capitales y empleos, pueden resumirse algunos efectos articuladores o desarticuladores de las economías de Norteamérica. Se aprecia mayor convergencia macroeconómica con relativa estabilidad económica y alineación plasmada en que la integración de infraestructuras carreteras y los flujos que circulan por ellas, lo que ha hecho aparecer ciclos de negocios paralelos al de Estados Unidos en México, con tasas de inflación e interés convergentes, pero eso ha causado debilitamiento de los encadenamientos productivos, desindustrialización y caída del poder adquisitivo de los sueldos y salarios en casi un 50 por ciento con respecto de los niveles de 1982, llevando a la desindustrialización. A esto se agrega la pérdida de soberanía y la deslegitimación del Estado nacional, que han tenido con-

a su destino final en Estados Unidos requiere de tres camiones diferentes además de los tiempos de espera en frontera" (De la Mora, 2011: 1, 2).

${ }^{14}$ En México, los costos logísticos los constituyen principalmente por costos de transporte; representan entre el 15 y el 20 por ciento del PIB, pero además, resultan entre el 50 y el 100 por ciento mayores que los del mercado de exportación más importante, Estados Unidos. 
secuencias sobre la identidad colectiva, el nacionalismo y la alineación del norte de México con el resto de Norteamérica

Entre los efectos del transporte transfronterizo del TLCAN, se puede destacar que, en efecto, el intercambio comercial aumentó doce veces, significativo porque su participación se incrementó del 38 al 65 por ciento del PIB en los últimos veinte años; sin embargo, también se verifican otros efectos como mayor asimetría en las capacidades nacionales de los tres países, que implica a su vez mayor desigualdad, exclusión, diferencias en el ritmo e intensidad del transporte, y empleo selectivo, observable en el incremento de la productividad del trabajo de los transportistas, paralela a la reducción de salarios y prestaciones.

\section{Conclusiones}

En la primera década del siglo XXI se completa la construcción de los grandes tramos de la infraestructura carretera que habían quedado pendientes, y es ahora cuando el Estado mexicano logra cumplir a cabalidad su función centralizadora territorial nacional -iniciada posiblemente en el gobierno de Lázaro Cárdenas-, pero ahora con un énfasis distinto, impuesto por la lógica global del capital, en el sentido de lograr competitividad internacional y alcanzar los estándares de conectividad, accesibilidad, velocidad y especificaciones carreteras y de complementariedad intermodal con otros transportes, para que la circulación física de mercancías y personas, capitales e informaciones, fluya desde y hacia México, junto con la del resto de Norteamérica.

En este contexto, las tendencias a la integración del transporte carretero y de las circulaciones en Norteamérica, de 1994 a 2014, sugieren que México es además de contrapeso territorial y reserva del subcontinente norteamericano, puente terrestre de grandes tráficos que circulan desde y hacia el Pacífico y Estados Unidos, por las ventajas de los significativos menores costos logísticos que esto representa. No obstante las ventajas comerciales que implica desarrollar esta vocación del territorio mexicano, el transporte terrestre y especialmente la libertad de tránsito de mercancías pactada con el Tratado desde 1994 siguen pendientes, trayendo consigo consecuencias desventajosas del proceso integrador y de la alineación comercial trinacional, que excluyen y atrasan de manera relativa a México aun más.

Si ya es un hecho el aumento del transporte transfronterizo por carretera, el cual representa más de dos terceras partes de los movimientos de carga internacionales y que ha sostenido el incremento comercial de doce veces lo exportado e importado por México, las fuerzas territoriales en juego están desatando procesos descontrolados que rompen cadenas productivas, vacían territorios y excluyen a localidades y mer- 
cados regionales porque no alcanzan las escalas e intensidades de los grandes tráficos. Esto plantea una reestructuración nacional de las regiones de México, un vuelco hacia el extranjero de la economía, crecientemente desindustrializada, que afecta la soberanía y coherencia interna, por las fuertes presiones extranjeras e internacionales.

Se ha mostrado cómo las mercancías transportadas por carreteras circulan libremente pero sólo a través de contenedores, ya que el resto de lo importado y exportado requiere de tres transferencias de carga que aumentan costos y demoras hasta un 30 por ciento más, lo que hace evidente que si bien con el TLCAN se ha incrementado doce veces el intercambio comercial internacional, esto no sucede a la velocidad requerida ni de manera libre ni equitativa para las partes contratantes de las operaciones comerciales, por lo que se ha constituido más bien en un tema pendiente que requiere revisarse a fondo, cotejando la vigencia y posibilidades de los objetivos iniciales contra los resultados y la situación actual. Sobre todo, con respecto de los efectos positivos y negativos que ha tenido sobre el comportamiento de las economías y los mercados mexicanos pero, de igual modo, sobre las ventajas comerciales y el bienestar de los actores, ciudades y regiones involucrados sin importar de las fronteras.

Aumenta el comercio, se reduce la soberanía y se profundizan desigualdades sin políticas públicas compensatorias. El transporte y las circulaciones comerciales de México quedan en posición más vulnerable y dependiente, se desatiende al mercado interno y no es fácil el aprovechamiento de las nuevas ventajas de la competitividad del transporte y la integración a nuevos y mayores mercados porque subsisten rupturas de carga y diferencias en los costos logísticos.

Por encima de las fracturas de carga, ocasionadas por la falta de continuidad de la infraestructura, y cambiando pero sin resolver a fondo las desigualdades en el acceso a las ciudades, puertos y regiones económicas, es muy probable que México ya se haya constituido en un espacio de intercambios comerciales continuos, pero la libertad de tránsito no se ha logrado, sólo existe para los contenedores de mercancías, quedando pendiente de cumplir el resto de lo acordado para el transporte y tránsito en el Capítulo xx del Tratado.

Así, aunque pudiera suponerse que el TLCAN cumplió sus cometidos comerciales, el transporte sigue siendo tema estratégico pendiente de resolver para lograr economías igualmente benéficas y no cada vez más desiguales entre los países, regiones y ciudades de Norteamérica. Subsisten retos que traspasan la vecindad geográfica, y cuya atención oportuna puede hacer trascender asimetrías territoriales, económicas y políticas, mucho más allá de las condiciones actuales de crisis, por medio de la cooperación y la equidad del desarrollo conjunto. 


\section{Biblografía}

\section{Camarena Luhrs, Margarita}

2013 "El Tratado de Libre Comercio: endeudamiento y costo soberano en México", Ola financiera no. 14, enero-abril, pp. 37-59, en <http: / / www.olafinanciera. unam.mx/new_web/14/pdfs / Camarena-Olafinanciera14.pdf>.

2012 “La circulación, espacio de la acción social”, Tecsistécatl vol. 4, no. 13, diciembre, pp. 1-13, en <http: / / www.eumed.net/ rev/ tecsistecatl/n13/ circulacionespacio-accion-social.html>.

2011 "Circulaciones irregulares o en redes, nueva geoestrategia en el contexto de Norteamérica”, Investigación social no. 11, invierno, pp. 1-30.

\section{Camarena Luhrs, Margarita y Lucio Flores Payán}

2013 "Economía financiera, infraestructura y costos de transporte de encadenamientos de valor en circulaciones completas de los 14 corredores de transporte del TLCAN en México", INCEPTUM vol. vIII, no. 14, enero-junio, pp. 137-160, en <file:/ / C:/Users/margaritacl/Downloads/inceptum\%202013\%20(4).pdf>.

De la Mora SÁnchez, Luz María

2011 "Transporte terrestre: el gran pendiente del TLCAN", Fortuna, pp. 1-3, en <http: / / revistafortuna.com.mx / contenido/2011/01/10/transporte-terrestre-el-gran-pendiente-del-tlcan/>.

\section{García Cano, Juan José Erazo y Manuel Cuan}

2010 "Estrategia de construcción y modernización de la red carretera nacional", ponencia presentada en la " 3 a Cumbre de las Américas 2020", Dallas, Texas, 12 de noviembre.

INEGi (INSTITUTO NACIONAL DE EsTADÍSTiCA y GEOGRAFía)

2011 “Cuéntame... Población”, en <http:/ / cuentame.inegi.org.mx>.

North American Transportation Statistics (nATs)

2011a "Cuadro 2", en Estadísticas de transporte de América del Norte, en <http: / / nats. sct.gob / nats / sys / tables.jsp?i=2\&id=2>.

2011b "Cuadro 15", en Estadísticas de transporte de América del Norte, en <http:/ / nats.sct.gob.mx/nats/sys / tables.jsp?i=2\&id=15>.

2011c "Cuadro 24", en Estadísticas de transporte de América del Norte, en <http:/ / nats.sct.gob.mx / nats / sys / tables.jsp?i=2\&id $=24>$. 
2011d "Cuadro 25", en Estadísticas de transporte de América del Norte, en <http:/ / nats.sct.gob.mx/nats / sys / tables.jsp?i=2\&id=25>.

2011e "Cuadro 26", en Estadísticas de transporte de América del Norte, en <http:/ / nats.sct.gob.mx / nats / sys / tables.jsp?i=2\&id=26>.

2011f "Cuadro 28", en <http:/ / nats.sct.gob.mx/nats/ sys / tables.jsp?i=2\&id=28>. 2011g “Cuadro 33", en <http:/ / nats.sct.gob/nats/sys/tables.jsp?i=2\&id=33>.

Ramírez Acosta, Ramón de Jesús y Ramón A. Castillo Ponce

2009 "Integración económica en América del Norte: lección de la experiencia de la Unión Europea para el TLCAN", Estudios fronterizos vol. 10, no. 19, enero-junio, pp. 1-13.

VOGEL, RiCHARD D.

2006 "The NAFTA Corridors: Offshoring U.S. Transportation Jobs to Mexico", Monthly Review vol. 57, no. 9, febrero, pp. 1-8, en <http: / / monthlyreview.org/2006/ $02 / 01$ / the-nafta-corridors-offshoring-u-s-transportation-jobs-to-mexico $>$. 